\title{
How much can inelastically scattered electrons contribute to electron cryotomography of biological specimens?
}

\author{
Joshua Dickerson ${ }^{1}$, Peng-Han $\mathrm{Lu}^{2}$, Dilyan Hristov ${ }^{3}$, Rafal Dunin-Borkowski ${ }^{4}$ and Christopher Russo ${ }^{5}$
}

${ }^{1}$ MRC Laboratory of Molecular Biology - University of Cambridge, Cambridge, England, United Kingdom, ${ }^{2}$ Ernst Ruska-Centre for Microscopy and Spectroscopy with Electrons and Peter Grünberg Institute, ForchungszentrumJülich, United States, ${ }^{3} \mathrm{MRC}$ Laboratory of Molecular Biology - University of Cambridge, United States, ${ }^{4}$ Forchungszentrum Jülich, Nordrhein-Westfalen, Germany, ${ }^{5}$ MRC Laboratory of Molecular Biology, United States

Electron cryotomography (cryoET) is an application of transmission electron cryomicroscopy (cryoEM) used to determine three-dimensional structures of cells and macromolecules [1]. The samples used for cryoET are often thicker than $1000 \AA$, meaning many $300 \mathrm{keV}$ electrons suffer at least one inelastic scattering event in transit through the specimen (Figure 1). Electrons that inelastically scatter have, by definition, lost significant energy and are thus incorrectly focused in the image due to the chromatic aberration $\left(C_{c}\right)$ in the objective lens. As a result, elastically scattered electrons that have also inelastically scattered do not provide high resolution information in phase contrast images and will instead contribute noise. The current practice is to remove these electrons with an electron energy filter to reduce the noise. Given the advent of practical chromatic aberration correction [2], there is a potential improvement in signal by incorporating these inelastically scattered electrons in the image in the context of an achromatic objective lens. Unfortunately, inelastic scattering not only changes the energy but also the direction of electrons, which can be thought of as reducing their spatial coherence. The aim of this study is to evaluate the conditions necessary to best use inelastically scattered electrons for biological imaging and determine the potential improvement in signal afforded by achromatic imaging of the thick biological specimens of interest in cryoET.

Ferrel used the Bohm-Pines electron plasma theory to derive the angular distribution of collective excitations [3], which are the source of most inelastic scattering in materials like amorphous water and carbon. The angular distribution is Lorentzian and can be approximated by the differential inelastic scattering cross section:

$$
\begin{aligned}
d \sigma_{\text {in }} & \propto \frac{1}{\theta_{E}^{2}+\theta^{2}} \text { with } \theta_{E}=\frac{\Delta p}{p} \begin{array}{l}
\text { where } \theta \text { is the angular deflection, } \Delta p \text { is the loss of momentum of } \\
\text { the inelastically scattered electron, and } p \text { is the magnitude of the } \\
\text { initial momentum. } \\
\text { The effect of the angular distribution on the signal can be }
\end{array}
\end{aligned}
$$
calculated by a spatial coherence envelope function, modified for a Lorentzian distribution instead of the standard Gaussian:

$$
\begin{aligned}
& E(\vec{k})=\int_{0}^{q_{c}} \frac{\gamma}{\left(q^{2}+\gamma^{2}\right)^{\frac{3}{2}}} J_{0}\left(2 \pi q\left(C_{s} \theta^{3}-\Delta z \theta\right)\right) q d q \\
& \sqrt{2 \gamma}=q_{c}
\end{aligned}
$$

where $q$ is the frequency, $q_{c}$ is the angular limit for plasmon scattering [4], $\gamma$ is the half width at half maximum for a Lorentzian distribution, $J_{0}$ is the zeroth order Bessel function, $\Delta z$ the defocus, and $C_{s}$ the spherical aberration coefficient.

To understand the loss of spatial coherence caused by the specimen, we experimentally determined the loss of signal from inelastically scattered electrons through a thick carbon specimen by measuring the fading of the 111 reflections from gold nanoparticles as a function of defocus. The results from these studies were confirmed by performing the same experiment on a $C_{c}$ corrected microscope (PICO) [5]. 
Simulations guided by the experimental data were then used to predict the rate of signal loss from inelastic scattering as a function of defocus (Figure 2).

The results show that inelastically scattered electrons can be used to enhance the signal in phase contrast images of thick specimens, provided that the sample is imaged within a few thousand Angströms of zero defocus. This indicates that a method of generating phase contrast, such as a phase plate, may be particularly useful with an achromatic objective lens. There will be a loss at high resolutions as a result of the defocus range over a thick specimen, and hence it is at lower resolutions (30-9 $\AA$ ) that there will be the most gain in signal. Energy filtering is also likely to be beneficial in combination with $C_{c}$ correction, albeit using a wider slit width, with the ideal width depending on the imaging conditions and desired resolution.

Overall, measurements using model carbon specimens indicate $C_{c}$ correction could increase the number of biological processes accessible to cryoET. More experiments with frozen biological specimen in a $C_{c}$ corrected cryomicroscope are needed to quantify such benefits.

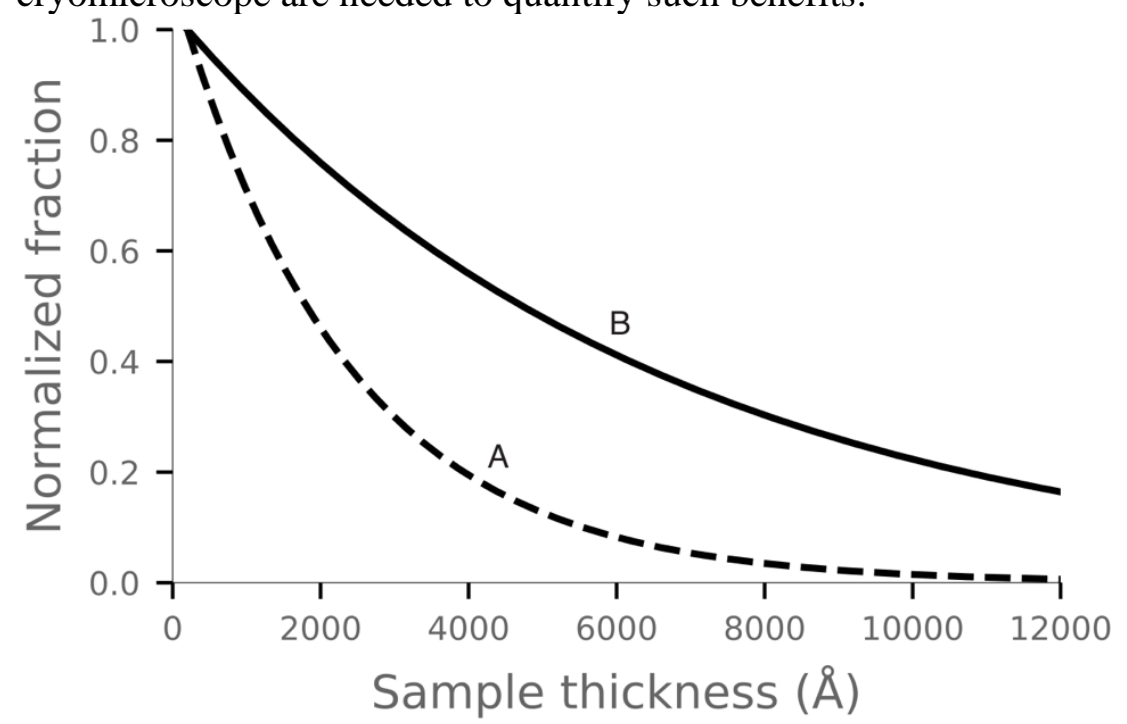

Figure 1.Comparison of signal as a function of specimen thickness for amorphous ice and accelerating voltage $300 \mathrm{kV}$. Plot A (dashed line) is the fraction of electrons that have undergone a single elastic scattering event and not been inelastically scattered, and plot B (solid line) also includes those that have inelastically scattered. The elastic scattering cross sections were taken from the ELSEPA database [6] and the inelastic scattering cross sections calculated from electron energy loss spectroscopy (EELS) measurements. The electron fractions were calculated using Poisson statistics and normalized to the value at $200 \AA$. 


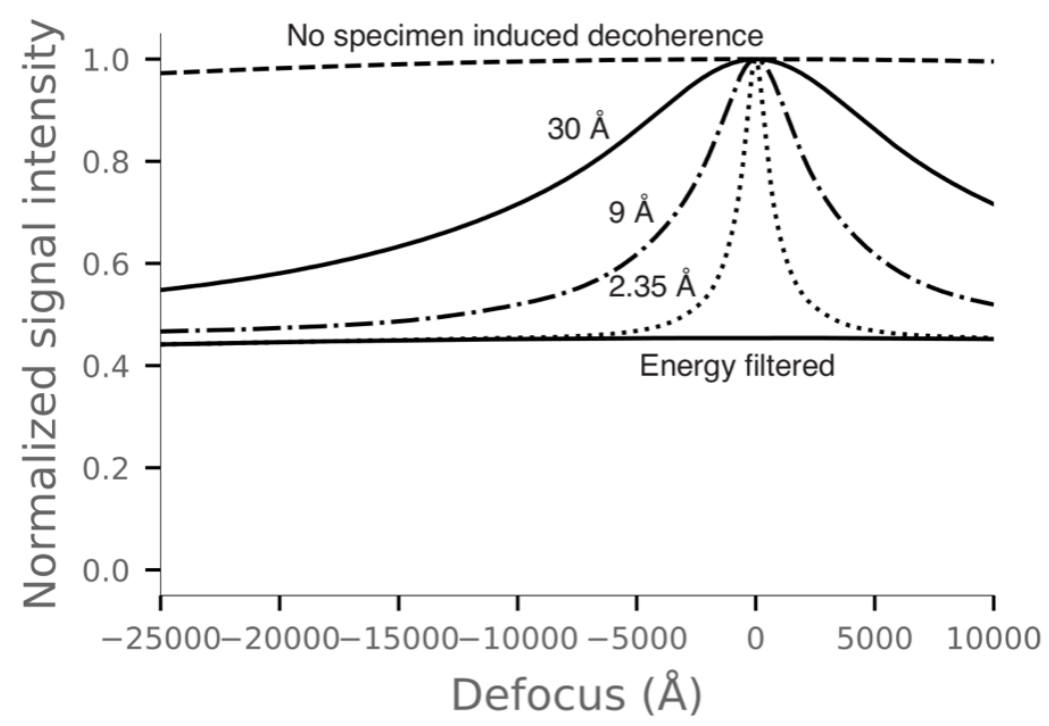

Figure 2.Plot of the simulated fading of power, as a function of defocus, for different resolutions from a sample of $3000 \AA$ thick amorphous water collected at $300 \mathrm{keV}$. The energy filter slit width is $10 \mathrm{eV}$. Note that this plot is for a microscope with the chromatic and spherical aberrations corrected, so the fading of power is symmetrical about 0 defocus.

\section{References}

[1] L. Gan, G. J. Jensen, Electron tomography of cells, Quarterly Reviews of Biophysics 45 (1) (2012) 2756. [2] B. Kabius, P. Hartel, M. Haider, H. Mller, S. Uhlemann, U. Loebau, J. Zach, H. Rose, First application of Cc-corrected imaging for high-resolution and energy-filtered TEM, Journal of Electron Microscopy 58 (3) (2009) 147-155.

[3] R. A. Ferrell, Angular dependence of the characteristic energy loss of electrons passing through metal foils, Phys. Rev. $101 \quad$ (1956) 554-563. doi:10.1103/PhysRev.101.554. URL https://link.aps.org/doi/10.1103/PhysRev.101.554

[4] R. H. Ritchie, Plasma losses by fast electrons in thin films, Phys. Rev. 106 (1957) 874-881. doi:10.1103/PhysRev.106.874.

[5] K. Tillmann, J. Barthel, L. Houben, FEI titan G3 50-300 PICO, Journal of large-scale research facilities JLSRF 1 (11 2015).

[6] F. Salvat, A. Jablonski, C. J. Powell, ELSEPA--Dirac partial-wave calculation of elastic scattering of electrons and positrons by atoms, positive ions and molecules, Computer Physics Communications 165 (2) (2005) $157-190$.

Acknowledgments

This work has received funding from the European Research Council (ERC) under the European Union's Horizon 2020 Research and Innovation Programme (Grant agreement No. 766970), the Medical Research Council grant MC_UP_120117, and the Herchel Smith PhD Scholarship. 